\title{
Nonbacterial Causes of Lymphangitis with Streaking
}

\author{
Brandon E. Cohen, MD, Arielle R. Nagler, MD, and Miriam Keltz Pomeranz, MD
}

Background: Lymphangitic streaking, characterized by linear erythema on the skin, is most commonly observed in the setting of bacterial infection. However, a number of nonbacterial causes can result in lymphangitic streaking. We sought to elucidate the nonbacterial causes of lymphangitic streaking that may mimic bacterial infection to broaden clinicians' differential diagnosis for patients presenting with lymphangitic streaking.

Methods: We performed a review of the literature, including all available reports pertaining to nonbacterial causes of lymphangitic streaking.

Results: Various nonbacterial causes can result in lymphangitic streaking, including viral and fungal infections, insect or spider bites, and iatrogenic etiologies.

Conclusion: Awareness of potential nonbacterial causes of superficial lymphangitis is important to avoid misdiagnosis and delay the administration of appropriate care. (J Am Board Fam Med 2016;29: 808-812.)

Keywords: Bacterial Infections; Diagnosis, Differential; Diagnostic Errors; Erythema; Iatrogenic Disease; Insects; Lymphangitis; MEDLINE; Mycoses; PubMed; Skin; Spider Bites

Lymphangitic streaking is characterized by linear erythema extending proximally toward regional lymph nodes, reflecting underlying inflammation of the superficial lymphatic vessels. Lymphangitic streaking is most often described in association with acute bacterial infections, such as Staphylococcus aureus, Streptococcus pyogenes, and Pasteurella multocida infections. ${ }^{1,2}$ Consequently, the presence of lymphangitic streaking often leads clinicians to treat patients with systemic antibiotics. ${ }^{2}$ However, superficial lymphangitis may also occur as a result of a variety of other nonbacterial etiologies, such as viral or fungal infections, arthropods bites, or iatrogenic interventions (Table 1).

The recognition of nonbacterial causes of lymphangitic streaking can be challenging. Accordingly, awareness of potential nonbacterial etiologies

This article was externally peer reviewed.

Submitted 8 January 2016; revised 19 May 2016; accepted 23 May 2016.

From the New York University School of Medicine, New York, NY (BEC); and the Ronald O. Perelman Department of Dermatology, New York University School of Medicine, New York (ARN, MKP).

Funding: none.

Conflict of interest: none declared.

Corresponding author: Miriam Keltz Pomeranz, MD, 240 E. 38th Street, 12th Fl., New York, NY 10016 (E-mail: Miriam.Pomeranz@nyumc.org). is important to avoid misdiagnosis, unnecessary antibiotic treatment, and a delay in the administration of appropriate treatments. Therefore we conducted a comprehensive review of the literature to characterize better the nonbacterial etiologies of lymphangitic streaking. All relevant English-language reports available through the PubMed/MEDLINE and Google Scholar databases were considered. Search terms included the terms lymphangitic streaking, superficial lymphangitis, or cutaneous infection, and one of the following terms: nonbacterial, viral, or fungal infection.

\section{Viral Infections}

Herpes simplex virus (HSV) has been reported to cause acute, superficial lymphangitis presenting with lymphangitic streaking. A number of reports of HSV of the upper extremity presenting with lymphatic streaking show no evidence of bacterial superinfection. ${ }^{3-7}$ Lymphangitic streaking associated with HSV may present along with systemic symptoms such as fever and lymphadenopathy (LAD), further mimicking bacterial infection. ${ }^{6,8} \mathrm{In}$ a number of the reported cases, patients were initially misdiagnosed with a bacterial infection and treated with systemic antibiotics. $4,7,8$ 
Table 1. Nonbacterial Causes of Lymphangitic Streaking

\begin{tabular}{ll}
\hline Viral & Herpes simplex \\
& Herpes zoster \\
Fungal & Aspergillus species \\
& Sporothrix schenckii \\
Arthropod bites & Insect bite \\
& Pigeon tick bite \\
& Spider bites \\
& Loxosceles reclusa (brown recluse spider) \\
& Loxosceles arizonica \\
Tuberculosis purified protein derivative & test \\
Iatrogenic & Bacillus Calmette-Guérin vaccine \\
& Cantharidin therapy \\
\hline
\end{tabular}

Gavelin and Knight $^{4}$ reported a case of a nurse who presented with tender, vesicular lesions of the right thumb, lymphangitic streaking, and axillary LAD. The patient was originally treated with erythromycin; however, culture revealed HSV without bacterial infection. Antibiotics were discontinued and the lesions resolved 11 days after the initial presentation. Dorman ${ }^{3}$ reported 2 patients presenting with recurrent herpes infection of the hand with superficial lymphangitis. Both patients had similar clinical presentations; however, 1 patient was found to have a bacterial superinfection requiring antibiotics, whereas the other patient was diagnosed with HSV infection alone, highlighting the diagnostic challenge. A patient recently presented at our institution with a recurrent, painful upper extremity bulla associated with a prominent lymphangitic streak. Initial treatment with levofloxacin was unsuccessful and bacterial culture was negative. Skin biopsy suggested occult herpesvirus infection versus a vesicular arthropod reaction. Clinically, herpes simplex infection was favored given the recurrent lesions on the same extremity, lack of insect exposure, history of oral herpes infection, and responsiveness to acyclovir; however, a final diagnosis could not be made with certainty (Figure 1).

Eruptions related to herpes zoster have also been described in association with lymphangitic streaking. ${ }^{7,9}$ However, clinical presentation and lesion distribution allow a diagnosis of herpes zoster to be more easily distinguished from bacterial lymphangitis compared with HSV. While superficial lymphangitis occurring in the setting of herpesvirus infections is well documented, potential pathogenic mechanisms have not been proposed.

\section{Fungal Infections}

The lymphocutaneous form of sporotrichosis typically manifests as nodular lymphangitis, characterized by the development of linear, erythematous lesions and subcutaneous nodules. ${ }^{10-14}$ However, there have been reports of patients presenting with lymphangitic streaks without nodules. ${ }^{15,16}$ In 1 such case, a pediatric patient presented with tender ulcers with raised borders and ascending lymphangitic streaks on the right upper extremity. The patient was treated with antibiotics, and symptoms worsened. Culture revealed infection with Sporothrix schenckii, and the patient was successfully managed on oral itraconazole. ${ }^{15}$

Lymphangitic streaking has also been associated with cutaneous Aspergillus infection. A 40-year-old man with acute lymphoblastic leukemia receiving chemotherapy presented with a tender, edematous fifth toe with lymphangitic streaks along the dorsal

Figure 1. Erythematous patch with central bulla on the ventral forearm, with a lymphangitic streak extending to the antecubital fossa in a patient with herpes simplex virus infection versus a reaction to an arthropod bite. (Photograph obtained by authors at New York University Langone Medical Center in January 2015.)

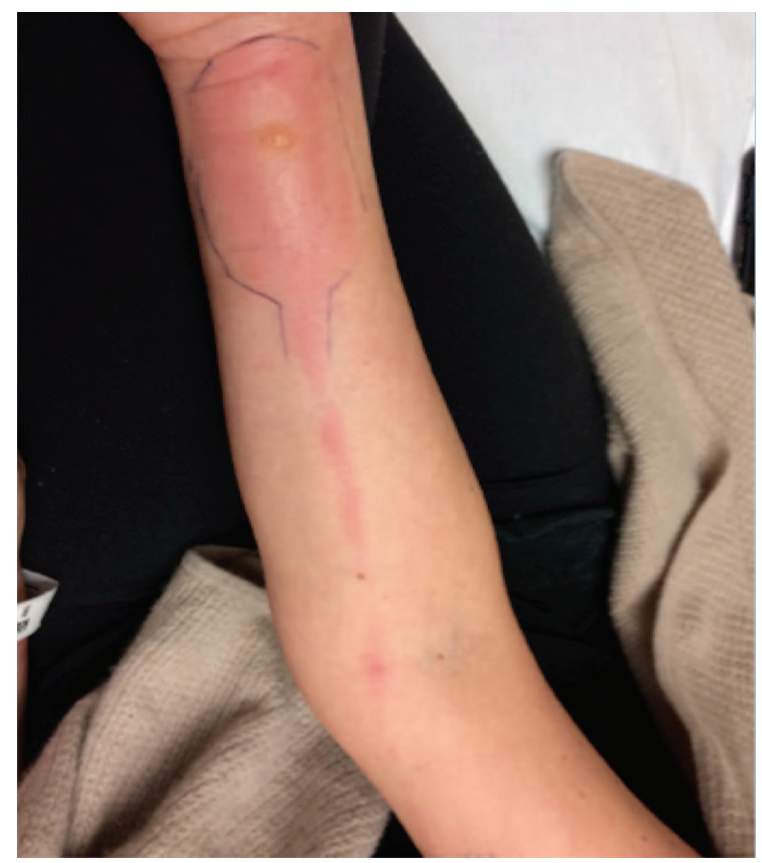


foot. Cultures revealed infection with an Aspergillus species without evidence of bacterial infection, and the patient was ultimately managed on amphotericin $\mathrm{B}$ and itraconazole. ${ }^{17}$

\section{Arthropod Bites}

Noninfectious lymphangitic streaking may also occur after bites from various insects, ticks, and spiders. ${ }^{2,18-22}$ In general, lymphangitic streaking associated with an insect bite has not been reported to present with systemic systems or LAD. ${ }^{2,18,20,21}$ The mechanism of lymphangitis is proposed to be secondary to either a hypersensitivity reaction or toxin injection. . $18,23,24$ Certain patient populations, such as those with hematologic abnormalities, have a greater tendency to develop exaggerated responses after arthropod bites. For instance, it has been well described that patients with chronic lymphocytic leukemia tend to develop exaggerated responses to arthropod bites, which manifest as vesicles or bullae with prominent edema and erythema that may be accompanied by lymphadenopathy and lymphangitic streaking ${ }^{25-27}$ (Figure 2). Patients with lymphangitic streaking associated

Figure 2. Two annular patches with surrounding erythema associated with lymphangitic streaks that developed after insect bites on the medial thigh of a patient with chronic lymphocytic leukemia.

(Photograph obtained by authors at New York University Langone Medical Center in July 2013.)

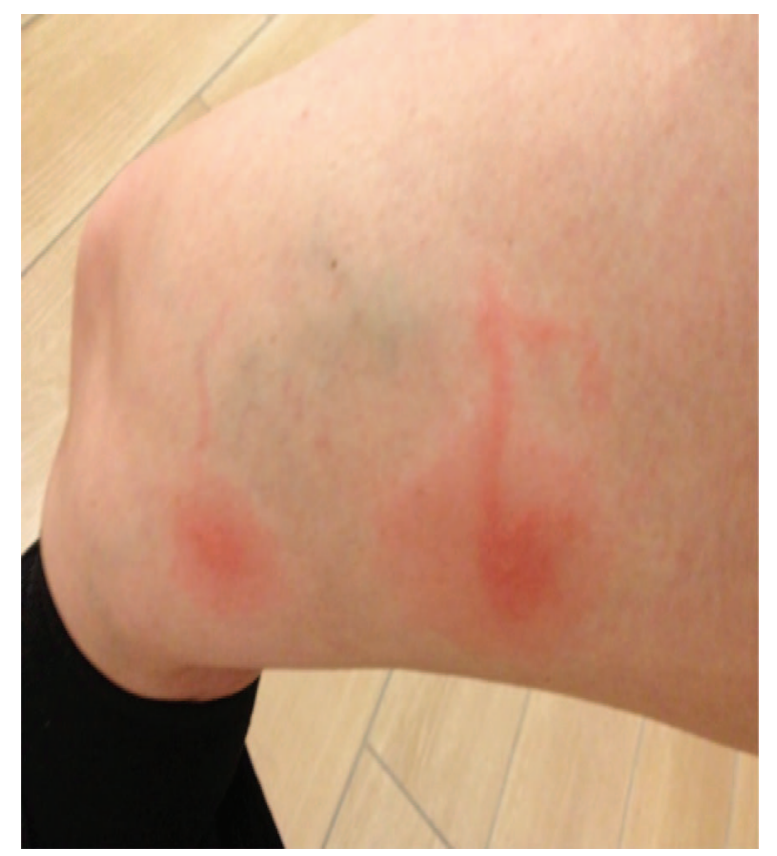

with an uncomplicated arthropod bite can be successfully managed with antihistamines and/or topical or oral steroids. ${ }^{20,23,24}$

In 1 case, a pediatric patient presented with prominent erythematous streaks extending from a lesion on the central abdomen toward the bilateral axilla, resembling a $V$ shape. Dermoscopy of the initial macule at the vertex of the lesion revealed a hemorrhagic center and crusted punctum, suggesting an insect bite. ${ }^{20} \mathrm{In}$ another case a patient presented with a pruritic lesion on the distal upper extremity that progressed to a red, tender linear streak extending toward the axilla. The pigeon tick (Argas reflexus) was recovered from the site and identified. The patient was treated with antihistamines and oral steroids, and the lesion resolved within 5 days. ${ }^{19}$

Spider bites, notably from those of Loxosceles species, are another cause of lymphangitic streaking that can occur without associated bacterial infection. Wright and colleagues ${ }^{22}$ performed a retrospective study of patients with suspected bites from Loxosceles reclusa, commonly known as the brown recluse spider. In this series, among 8 patients who required hospitalization, 2 presented with lymphangitic streaking extending from the location of the bite. In another case a pediatric patient presented with a bite on the abdomen from the brown spider Loxosceles arizonica. Lymphangitic streaks were observed extending from the bite superiorly toward the right axilla and inferiorly to the right inguinal area. Systemic systems and LAD were absent. The patient was successfully managed with a 4-day course of prednisone. ${ }^{23}$

\section{Iatrogenic Causes}

Several reported noninfectious, iatrogenic causes of lymphangitic streaking have been associated with vaccinations, purified protein derivative (PPD) placement, and treatment with cantharidin. In 1 case series, 12 patients developed superficial lymphangitis after PPD placement for tuberculosis testing. Patients developed systemic systems and cutaneous lesions as early as several hours after PPD placement. ${ }^{28}$ Similar reports exist after administration of the Bacillus Calmette-Guérin vaccine; these patients presented with lymphangitic streaking extending from the vaccination site to the supraclavicular or cervical lymph nodes. ${ }^{29}$

Lymphangitic streaking has also been reported after topical application of cantharidin for verrucae 
vulgaris. ${ }^{30,31}$ In 1 case series, 2 patients developed edema and tender lymphangitic streaks on the extremities 1 day after the application of topical cantharidin. ${ }^{30}$ It was suggested the lymphangitis may be related to an inflammatory reaction extending to the lymphatic vessels. ${ }^{31}$

\section{Conclusion}

Lymphangitic streaking has a number of nonbacterial etiologies, including viral and fungal infections and reactions to insect or spider bites. In addition, several episodes of lymphangitic reactions to medical interventions in the absence of bacterial superinfection have been reported. Because several nonbacterial etiologies of lymphangitic streaking can mimic bacterial infection, it can present a diagnostic challenge. It is important to include alternative etiologies in the differential diagnosis of patients with lymphangitic streaking, particularly those patients who do not respond to antibacterial therapy. Awareness of the differential diagnosis of lymphangitic streaking may help reduce the incidence of misdiagnosis and promote appropriate patient management.

\section{References}

1. Kano Y, Inaoka M, Shiohara T. Superficial lymphangitis with interface dermatitis occurring shortly after a minor injury: possible involvement of a bacterial infection and contact allergens. Dermatology 2001;203:217-20.

2. Marque M, Girard C, Guillot B, Bessis D. Superficial lymphangitis after arthropod bite: a distinctive but underrecognized entity? Dermatology 2008;217: 262-7.

3. Dorman JM. Herpetic lymphangitis in a student population. J Adolesc Health Care 1982;3:49-50.

4. Gavelin GE, Knight CR. Herpes simplex infection of the finger. CMAJ 1965;93:366-7.

5. Howard WR, Taylor JS, Steck WD. Lymphatic complications of manual herpes simplex infection. Cutis 1979;23:580-3.

6. Sands M, Brown R. Herpes simplex lymphangitis. Two cases and a review of the literature. Arch Intern Med 1988;148:2066-7.

7. Trice ER, Shafer JC. Recurrent herpes simplex infections of upper extremities with lymphangitis; report of three cases with virus studies in two. AMA Arch Derm Syphilol 1953;67:37-41.

8. Ward JR, Clark L. Primary herpes simplex virus infection of the fingers. JAMA 1961;176:226-8.

9. Nicolau S, Poincloux P. Etude clinique et expérimentale d'un cas d'herpès récidivant du doigt. Ann Inst Pasteur 1924;38:977.
10. Crevasse L, Ellner PD. An outbreak of sporotrichosis in Florida. JAMA 1960;173:29-33.

11. Kostman JR, DiNubile MJ. Nodular lymphangitis: a distinctive but often unrecognized syndrome. Ann Intern Med 1993;118:883-8.

12. Ryncarz R, Heasley EC, Babinchak TJ. The clinical spectrum of nodular lymphangitis. Hosp Physician 1999;9:63-6.

13. Tobin EH, Jih WW. Sporotrichoid lymphocutaneous infections: etiology, diagnosis and therapy. Am Fam Physician 2001;63:326-32.

14. Foerster H. Sporotrichosis, an occupational dermatosis. JAMA 1926;87:1605-9.

15. Howe WR, Wisco OJ, Sartori C. Fixed cutaneous sporotrichosis in an adolescent boy: a case report. Cutis 2006;78:337-40.

16. Naqvi SH, Becherer P, Gudipati S. Ketoconazole treatment of a family with zoonotic sporotrichosis. Scand J Infect Dis 1993;25:543-5.

17. Brodsky JW, Seidenfeld SM, Brooks B, Shabat S. Aspergillus osteomyelitis and lymphangitis in immunocompromised patient after toenail clipping. Foot Ankle Int 2005;26:576-8.

18. Abraham S, Tschanz C, Krischer J, Saurat JH. Lymphangitis due to insect sting. Dermatology 2007;215: 260-1.

19. Koul PA, Qadri SM. Acute superficial lymphangitis following pigeon mite bite. BMJ Case Rep 2010;2010. pii. bcr0920103310. doi: 10/1136/bcr.09.2010.3310.

20. Piccolo V, Russo T, Picciocchi R, Diplomatico M, Ametrano O, Moscarella E. Superficial lymphangitis after insect bite. J Pediatr 2013;163:299.e1.

21. Smith LR, Honig PJ. Lymphangitis of the anterior chest wall. Cutis 1980;26:412-3.

22. Wright SW, Wrenn KD, Murray L, Seger D. Clinical presentation and outcome of brown recluse spider bite. Ann Emerg Med 1997;30:28-32.

23. Knutson CA, Cohen M, Hansen RC. Spider bite with lymphangitic streaking. Brown or violin spider bite with atypical features. Arch Dermatol 1992;128: 255-6, 258-9.

24. Maso MJ, Lin RY, Bagley MP, Cohen PJ, Schwartz RA. Immunopathological changes after multiple spider bites. Contact Dermatitis 1987;17:65-8.

25. Asakura K, Kizaki M, Ikeda Y. Exaggerated cutaneous response to mosquito bites in a patient with chronic lymphocytic leukemia. Int J Hematol 2004; 80:59-61.

26. Davis MD, Perniciaro C, Dahl PR, Randle HW, McEvoy MT, Leiferman KM. Exaggerated arthropod-bite lesions in patients with chronic lymphocytic leukemia: a clinical, histopathologic, and immunopathologic study of eight patients. J Am Acad Dermatol 1998;39:27-35.

27. Pedersen J, Carganello J, van der Weyden MB. Exaggerated reaction to insect bites in patients with 
chronic lymphocytic leukemia. Clinical and histological findings. Pathology 1990;22:141-3.

28. Morrison JB. Lymphangitis after tuberculin tests. $\mathrm{Br}$ Med J (Clin Res Ed) 1984;289:413.

29. Hartston W. Uncommon skin reactions after BCG vaccination. Tubercle 1959;40:265-70.
30. Dilaimy M. Letter: Lymphangitis caused by cantharidin. Arch Dermatol 1975;111:1073.

31. Stazzone AM, Borgs P, Witte CL, Witte MH. Lymphangitis and refractory lymphedema after treatment with topical cantharidin. Arch Dermatol 1998;134: 104-6. 